\title{
SCALING CONTRIBUTIONS TO THE FREE ENERGY IN THE 1/N EXPANSION OF O(N) NONLINEAR SIGMA MODELS IN d-DIMENSIONS
}

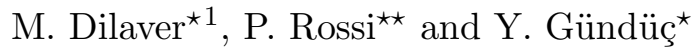 \\ *Hacettepe University, Physics Department, 06532 Beytepe, Ankara, Turkey. \\ ${ }^{\star \star}$ Dipartimento di Fisica dell’Universita and INFN , I-56100 Pisa, Italy.
}

\begin{abstract}
.
Within the $1 / N$ expansion of $O(N)$ nonlinear $\sigma$ models for $d \leq 4$ it is possible to separate consistently the spin-wave and the massive-mode contributions to the scaling part of the free energy near criticality, and to evaluate them to $O(1 / N)$. For critical dimensions $d=2+2 / n$ the Abe-Hikami anomaly is recovered, while for $d=2$ the removal of the spin-wave term is justified.
\end{abstract}

\footnotetext{
${ }^{1}$ Hacettepe University, Physics Department, 06532, Beytepe, Ankara, Turkey fax: + (90) 312 2352550; e-mail: dilaver@thep1.phys.hun.edu.tr
} 
Scaling is a fundamental property of physical systems in the neighborhood of a second order phase transition. In the field theory approach to the study of critical phenomena it is easy to identify the scaling properties of correlation functions. However it may be difficult to study scaling of bulk thermodynamical properties, and in particular the free energy. The reason behind this is the phenomenon of mixing: in a renormalizable field theory the vacuum expectation values of composite operators require in general not only a multiplicative renormalization, but also the subtraction of the contributions coming from lower-dimensionality operators carrying the same quantum numbers. These contributions, when present, are numerically dominant with respect to the scaling part of the expectation value, for the very simple reason that they are associated with lower powers of the (vanishing) mass scale, or inverse correlation length. Therefore one must in principle compute these terms with infinite accuracy (summing infinite orders of perturbation theory) in order for the evaluation of scaling contributions to become possible. In practice this "subtraction of perturbative tails" has been attempted with some success even in purely numerical computations of topological susceptibilities 11. The reason behind this possibility stays in the fact that the physical degrees of fredom generating these contributions, the "spin waves", are quite different in nature from the (massive, topological) degrees of freedom associated with the scaling terms. Therefore, in a numerical simulation based on heating, the spin waves can be excited quite independently and earlier than the massive fields. When a plateau is temporarily reached, the value of the plateau can be nonperturbatively identified with the spin wave contribution, to be subtracted from the final (fully thermalized) vacuum expectation value.

There are obvious conceptual and technical limits in the accuracy of this determination, and it would be nice to possess some analytical nonperturbative scheme in order to deal with this problem. In the context of two-dimensional $O(N)$ lattice spin models, and of their regularized continuum counterparts, it was noticed some time ago that the $1 / N$ expansion offered the possibility of consistently defining the "perturbative tails" of the free and internal energies, at least up to second nontrivial order [2]. The procedure adopted might however appear to be tailored upon the 
two-dimensional case, where criticality occurs in the zero-coupling limit, the models are perturbatively renormalizable and asymptotically free, and the whole procedure essentially amounted to a prescription (principal value integration) for the evaluation of the integral representing the resummation of an infinite series of perturbative Feynman diagrams. We therefore decided to consider the problem of evaluating the scaling part of the free energy in $O(N)$ nonlinear sigma models around criticality in dimensions $2<d<4$, where criticality occurs at a finite value of the lattice coupling, and the critical exponents (hence the scaling properties) are nontrivial.

Finding a subtraction procedure allowing for a nonambiguous identification of the scaling contributions cannot in this case be considered simply as the problem of making sense out of the formal sum of a known perturbative series, since we must first face the problem of correctly identifying which contributions come from spin wave degrees of freedom.

For definiteness, let's consider the continuum version of nonlinear sigma models:

$$
\mathcal{S}=\frac{1}{2} N \beta \int d^{d} x \partial_{\mu} \overrightarrow{\mathbf{S}} \cdot \partial_{\mu} \overrightarrow{\mathbf{S}}
$$

We label the coefficents of the $1 / N$ expansion for any physical quantity $Q$ according to the notation:

$$
Q=\sum_{i} Q_{i} N^{-i}
$$

The (unsubtracted) free energy of the above model can be computed in the $1 / N$ expansion and its formal expression is ;

$$
F=\frac{N}{2} \int \frac{d^{d} p}{(2 \pi)^{d}} \ln \beta\left(p^{2}+m_{0}^{2}\right)-\frac{N}{2} \beta m_{0}^{2}+\frac{1}{2} \int \frac{d^{d} p}{(2 \pi)^{d}} \ln \left[\Delta^{-1}\left(p, m_{0}\right)\right]+O(1 / N)
$$

where $m_{0}$ is defined by the gap equation:

$$
\beta=\int \frac{d^{d} p}{(2 \pi)^{d}} \frac{1}{p^{2}+m_{0}^{2}}
$$

see ref [3] for explainations and notation. 
We introduced the inverse propagator of the effective field:

$$
\begin{aligned}
\Delta^{-1}\left(p, m_{0}\right) & =\frac{1}{2} \int \frac{d^{d} p}{(2 \pi)^{d}} \frac{1}{q^{2}+m_{0}^{2}} \frac{1}{(p+q)^{2}+m_{0}^{2}} \\
& =\frac{\Gamma(2-d / 2)}{2(4 \pi)^{d / 2}}\left(\frac{p^{2}}{4}+m_{0}^{2}\right)^{d / 2-2}{ }_{2} F_{1}\left(2-\frac{d}{2}, \frac{1}{2} ; \frac{3}{2} ; \frac{p^{2}}{p^{2}+m_{0}^{2}}\right)
\end{aligned}
$$

where ${ }_{2} F_{1}\left(2-\frac{d}{2}, \frac{1}{2} ; \frac{3}{2} ; \frac{p^{2}}{p^{2}+m_{0}^{2}}\right)$ is hypergeometric function 此.

It will be essential to our analysis that the inverse propagator admits an asymptotic expansion for small $m_{0}$ [5, 6], which is easily extracted from the representation:

$$
\Delta^{-1}\left(p, m_{0}\right)=\frac{\Gamma(2-d / 2)}{2(4 \pi)^{d / 2}} \frac{\Gamma(d / 2-1)^{2}}{\Gamma(d-2) p}\left(p^{2}+4 m_{0}^{2}\right)^{\frac{d-3}{2}}+\frac{\left(\beta-\beta_{c}\right)}{p^{2}+4 m_{0}^{2}}{ }_{2} F_{1}\left(\frac{d-1}{2}, 1 ; \frac{d}{2} ; \frac{1}{1+\frac{p^{2}}{4 m_{0}^{2}}}\right)
$$

where $\beta_{c}$ is the (scheme-dependent) critical value of the coupling and one can show that, for small $m_{0}$

$$
\left(\beta-\beta_{c}\right)=\frac{\Gamma(1-d / 2)}{(4 \pi)^{d / 2}}\left(m_{0}^{2}\right)^{d / 2-1}
$$

Such an asymptotic expansion can in turn be reinterpreted as the effect of an operator expansion. In order to identify the spin-wave contribution to the free energy we must evaluate the small-mass limit of eq.(3):

$$
F^{s w}=\frac{N}{2} \int \frac{d^{d} p}{(2 \pi)^{d}} \ln \left(\beta p^{2}\right)+\frac{1}{2} \int \frac{d^{d} p}{(2 \pi)^{d}} \ln \Delta_{0}^{-1}\left(p, m_{0}\right)+O(1 / N)
$$

where from eq.(6) we extracted the definition :

$$
\Delta_{0}^{-1}\left(p, m_{0}\right)=\frac{\Gamma(2-d / 2) \Gamma(d / 2-1)^{2}}{2(4 \pi)^{d / 2} \Gamma(d-2)} p^{d-4}+\frac{\Gamma(1-d / 2)}{(4 \pi)^{d / 2}} \frac{m_{0}^{d-2}}{p^{2}}
$$

The definitions eq.(8) and eq.(9) were given with the aim of isolating those contributions to the free energy that are originated from a mixing with the identity operator. In order to justify our choice we must however prove that the subtracted free energy possesses the correct scaling properties. 
This is achieved by proving finiteness and scheme-independence of the dimensionless ratio;

$$
f=\frac{1}{N}\left(F-F^{(s w)}\right) m^{-d}
$$

where we have introduced the mass gap $m^{2}$ (inverse correlation length), whose large $N$ limit is $m_{0}^{2}$. It is easy to obtain the $O(1 / N)$ correction in the form:

$$
m_{1}^{2}=\int \frac{d^{d} p}{(2 \pi)^{d}} \frac{\Delta\left(p, m_{0}\right)}{\left(p+i m_{0}\right)^{2}+m_{0}^{2}}+\frac{1}{2} \Delta(0) \int \frac{d^{d} p}{(2 \pi)^{d}} \Delta\left(p, m_{0}\right) \frac{d}{d m_{0}^{2}} \Delta^{-1}\left(p, m_{0}\right)
$$

The ratio $f$ can also be expanded in powers of $1 / N$. The calculation of $f_{0}$ is straight-forward. The result is scheme-independent, and it is most easily derived by the use of dimensional regularization:

$$
f_{0}=\frac{1}{2}\left[\int \frac{d^{d} p}{(2 \pi)^{d}} \ln \left(1+\frac{m_{0}^{2}}{p^{2}}\right)-\beta m_{0}^{2}\right] m_{0}^{-d}=\frac{2}{d} \Delta^{-1}\left(0, m_{0}\right) m_{0}^{4-d}
$$

The value of $f_{1}$ can be obtained from:

$$
f_{1}=\frac{1}{2}\left[\int \frac{d^{d} p}{(2 \pi)^{d}} \ln \left[\Delta_{0}\left(p, m_{0}\right) \Delta^{-1}\left(p, m_{0}\right)\right] m_{0}^{-d}-\frac{d}{2} f_{0} \frac{m_{1}^{2}}{m_{0}^{2}}\right.
$$

In order to find a convenient representation for $f_{1}$, we make the observation that the total derivative with respect to $m_{0}^{2}$ can be represented in terms of the partial derivatives according to:

$$
\frac{d}{d m_{0}^{2}}=\frac{\partial}{\partial m_{0}^{2}}+\frac{d \beta}{d m_{0}^{2}} \frac{\partial}{\partial \beta}=\frac{\partial}{\partial m_{0}^{2}}-2 \Delta^{-1}\left(0, m_{0}\right) \frac{\partial}{\partial \beta}
$$

Moreover the following identity holds under symmetric integration:

$$
\frac{1}{\left(p+i m_{0}\right)^{2}+m_{0}^{2}} \rightarrow \frac{\partial}{\partial \beta} \Delta^{-1}\left(p, m_{0}\right)=\frac{1}{p^{2}+4 m_{0}^{2}}{ }_{2} F_{1}\left(\frac{d-1}{2}, 1 ; \frac{d}{2} ; \frac{1}{1+\frac{p^{2}}{4 m_{0}^{2}}}\right)
$$

implying that:

$$
m_{1}^{2}=\frac{1}{2} \Delta\left(0, m_{0}\right) \int \frac{d^{d} p}{(2 \pi)^{d}} \Delta\left(p, m_{0}\right) \frac{\partial}{\partial m_{0}^{2}} \Delta^{-1}\left(p, m_{0}\right)
$$

and as a consequence: 


$$
f_{1}=\frac{1}{2} m_{0}^{-d}\left\{\int \frac{d^{d} p}{(2 \pi)^{d}}\left[\ln \Delta^{-1}\left(p, m_{0}\right)-\ln \Delta_{0}^{-1}\left(p, m_{0}\right)-m_{0}^{2} \frac{\partial}{\partial m_{0}^{2}} \ln \Delta^{-1}\left(p, m_{0}\right)\right]\right\}
$$

We recognize that the expression appearing in the integrand of eq. 117) must be $O\left(m_{0}^{4}\right)$, hence by naive power counting, confirmed by detailed analysis, the large-momentum behavior of the integrand turns out to be $O\left(1 / p^{4}\right)$. This confirms our statement that $f_{1}$ is finite for all $d<4$, independent of the regularization scheme adopted.

Let's notice that $f$ was evaluated for $d=2$ in ref [2]; and the result was: $f_{0}=\frac{1}{8 \pi}, f_{1}=-2 f_{0}$.

For $d=3$ we obtained: $f_{0}=\frac{1}{24 \pi}$

$$
\begin{aligned}
\frac{f_{1}}{f_{0}} & =\frac{6}{\pi} \int_{0}^{\infty} x^{2} d x\left[\ln \left(\frac{2}{\pi} \arctan \frac{x}{2}\right)-\ln \left|1-\frac{4}{\pi x}\right|-\frac{1}{\arctan \frac{x}{2}}\left(\frac{1}{2} \arctan \frac{2}{x}-\frac{x}{4+x^{2}}\right)\right] \\
& \cong 1.97863
\end{aligned}
$$

Since for all $d>1 \Delta_{0}^{-1}$ vanishes for some real positive value of $p^{2}$, we stress that our integration procedure must always be specified by the request that no imaginary part should appear in the final result (principal value prescription ) [6]. Our results for the range $1 \leq d \leq 4$ are given by Figure 1.

In order for our analysis to be complete, we must remember that in the critical regime even the high-frequency modes (spin waves) can bring a scaling contribution to the free energy [7]. While logically distinct from the quantity we have just computed in the context of quantum field theories where a normal-ordering procedure can be defined in order to remove such a contribution, the scaling free energy originated from spin waves cannot be isolated in statistical systems and numerical simulations. Hence it is important to be able to compute the term proportional to $m^{d}$ in eq.(8). This is actually a reasonably simple task, since we can now exploit the properties of dimensional regularization and evaluate analytically eq.(8) for arbitrary $d<1$

From the explicit representation of $\Delta_{0}^{-1}\left(p, m_{0}\right)$, eq.(9), we obtain in dimensional regularization: 


$$
\begin{aligned}
F^{(s w)} & =\frac{1}{2} \int \frac{d^{d} p}{(2 \pi)^{d}} \ln \left[p^{d-2}+\frac{4}{2-d} \frac{\Gamma(d-2)}{\Gamma(d / 2-2)^{2}} m_{0}^{d-2}\right] \\
& =\alpha_{1} f_{0} m_{0}^{d} \frac{1}{2(d-1)}\left[\frac{4}{2-d} \frac{\Gamma(d-2)}{\Gamma(d / 2-1)^{2}}\right]^{\frac{2}{d-2}} \Gamma\left(1-\frac{2}{2-d}\right) \Gamma\left(\frac{2}{2-d}\right)
\end{aligned}
$$

where we have introduced the $O(1 / N)$ coefficient $\alpha_{1}$ in the expansion of the critical exponent of the specific heat:

$$
\alpha_{0}=\frac{d-4}{d-2} \quad \alpha_{1}=\frac{2 \Gamma(d)}{\Gamma(2-d / 2) \Gamma(d / 2)^{3}}
$$

It is important to notice that the imaginary part of the r.h.s. of eq. (19) is exactly needed in order to cancel the imaginary part originated by the naive integration of eq.(17), which further justifies the procedure of removing all imaginary contributions.

Here we face the phenomenon known as Abe-Hikami anomaly $[8]$; whenever $2 /(d-2)$ becomes a positive integer $n$, the expression in eq.(19) is singular. However because of eq.(7), in that same circumstance the expression we obtained is proportional to the positive integer power $n+1$ of $\beta-\beta_{c}$. We can therefore subtract a term analytic in $\beta$ from eq.(19) and obtain the scaling contribution of spin waves to the free energy in the form:

$$
F_{s u b}^{(s w)}=\frac{N f_{0} m_{0}^{d}}{2(d-1)}\left[\frac{4}{d-2} \frac{\Gamma(d-2)}{\Gamma(d / 2-1)^{2}}\right]^{\frac{2}{d-2}}+O(1)
$$

The most interesting feature of this result is the dependence on $N$, implying that for the special values $d=2+2 / n$ even the large $N$ limit of the free energy is affected by the spin-wave contribution. When $d \rightarrow 2$, corresponding to $n \rightarrow \infty$, singularities of the r.h.s. of eq.(20) accumulate on both sides of the real axis in the complex $d$ plane. However it is easy to check that the limit exists when a small imaginary part is added to $d$, and its value is consistently 0 , which further justifies the procedure of removing all spin-wave contributions in the two-dimensional case.

The support from TÜBİTAK is acknowledged. 


\section{References}

[1] A. Di Giacomo and E. Vicari, Phys. Lett. B 275 (1992) 429.

[2] M. Campostrini and P. Rossi, Phys. Lett. B 242 (1990) 81.

[3] P. Biscari, M. Campostrini and P. Rossi, Phys. Lett. B 242 (1990) 225

[4] I. S. Gradshteyn and I. M. Ryzhik: Table of Integrals, Series and Products (Academic Press, London, 1980)

[5] V. F. Muller and W. Ruhl, Ann. Phys. (NY) 168 (1986) 425.

[6] M. Campostrini and P. Rossi, Riv. Nouvo Cim. 16 n.6 (1993) 1.

[7] S. Ma, Journ. Math. Phys. 15 (1974) 1866.

[8] R. Abe and S. Hikami, Prog. Teor. Phys. 51 (1974) 1041. 
FIGURE CAPTIONS

Figure 1. $f_{1} / f_{0}$ versus d. 


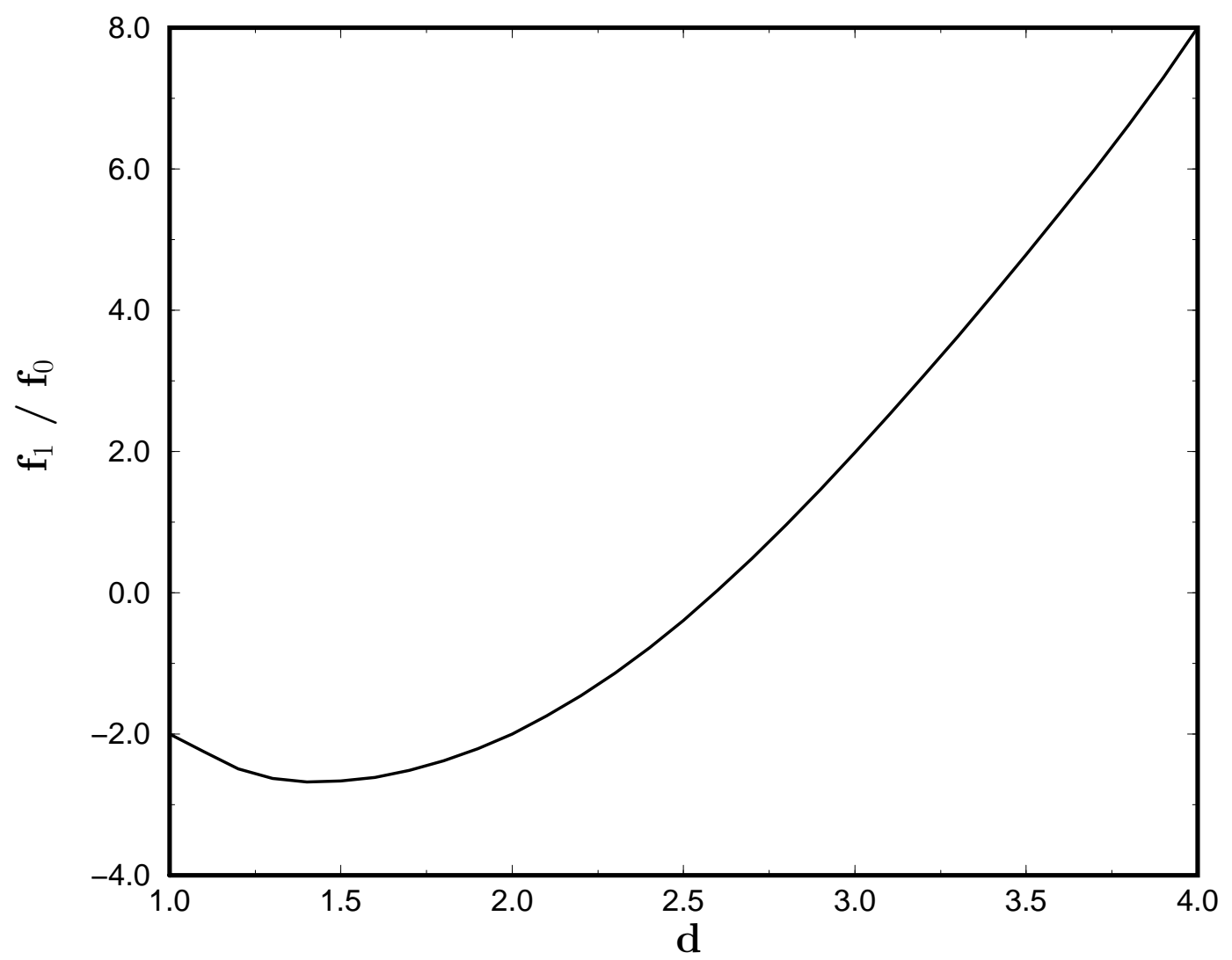

Figure 1: 